\title{
A Conversational Agent for Social Support: Validation of Supportive Dialogue Sequences
}

\author{
Janneke M. van der Zwaan, Virginia Dignum, and Catholijn M. Jonker \\ Delft University of Technology
}

\begin{abstract}
Recently, we proposed a dialogue model for social support. To validate this model, we analyzed 23 real world chat conversations. After some adjustments, the dialogue sequence patterns specified in the model cover $87.4 \%$ of the data. Based on this result, we conclude that the dialogue model accurately describes comforting conversations. Next, the model will be incorporated into a comforting ECA.
\end{abstract}

\section{Introduction}

Social support or comforting refers to all communicative attempts to alleviate the emotional distress of another person [2]. In our research, we are exploring how and to what extent Embodied Conversational Agents (ECAs) can provide social support. Recently, we proposed a domain-independent dialogue model to provide social support in response to upsetting events such as bullying [4]. In particular, the model specifies dialogue sequences that allows an ECA to verbally express social support [3]. So far, it was unclear to what extent these sequences occur in actual comforting dialogues. To assess the validity of our model, we analyzed dialogue sequences in real comforting conversations about bullying.

\section{Dialogue Model for Social Support}

In the dialogue model, a comforting conversation consists of 5 phases (cf. the 5-phase model [1]): 1) Welcome; 2) Determine the user's situation; 3) Determine conversation objective (e.g., getting tips on how to deal with bullying); 4) Give advice; and 5) Round up. For communicating social support, phase 2 (Determine situation) and 4 (Give advice) are the most important phases. Therefore, only the sequences in these phases have been analyzed.

Conversation phases consist of one or more dialogue sequences. A dialogue sequence is a set of utterances (conversation turns) in which a request for information or the proactive sharing of a piece of information is completed by the dialogue partners. Phase 2 consists of a recurring pattern of the agent asking a question, the user answering that question and the agent acknowledging the answer. Optionally, the agent expresses support by giving a sympathetic remark, compliment or encouragement to the user (pattern QA). In phase 4, the agent asks the user his plans on how to deal with the situation; this topic is discussed 
using sequence pattern QA. Additionally, the agent proactively utters advice and the user confirms that advice (pattern Advice). Optionally, a piece of advice is followed by a list of instructions (pattern Teaching). More details about the sequence patterns can be found in [3].

\section{Data Analysis}

Twenty-three real world chat conversations about bullying were analyzed. The data was anonymized and consisted of the counselor's utterances and the positions of the user's utterances. After dividing the conversations into the five phases, phases 2 and 4 were divided into sequences of utterances. Next, patterns occurring in the sequences were extracted. We started with the sequences as specified in the dialogue model and adjusted or added patterns when needed.

Because the user utterances were unavailable in the data, the contents of a user turn are determined based on the response of the counselor. User utterances were included patterns only if the counselor explicitly responded to them.

\section{Results}

Analysis of the sequences in the corpus suggested that the sequence specifications were too strict. Therefore, two out of three sequences specified in the model have been adjusted to better fit the data. In total, the data contained 10 different sequence patterns. After adjustment, the patterns specified by the model cover $87.4 \%$ of the sequences. Due to space constraints, we only discuss how the sequence patterns were adjusted and present the most frequent new pattern.

In the QA pattern, the agent's acknowledgment was made optional, because counselors do not always respond to a user's answer. Additionally, users did not seem to confirm the counselor's advice, so the user confirmation in the Advice sequence was made optional as well. While the model specifies a direct way of giving advice (the counselor/agent telling the user what to do), the data contains more indirect styles for giving advice. After giving advice the counselor requests feedback, e.g. 'You can talk to a teacher. How about that?' (C46) and optionally expresses support after a user responds to a feedback request.

The most important new sequence pattern found in the data can be characterized as the counselor responding to information the user proactively introduces

during the conversation. This pattern RtU (Respond to User) accounts for $5.6 \%$ of the sequences that occur in phase 2 and 4 .

\section{Conclusion}

Our analysis shows that there are many regularities in the data. While we had to adjust the proposed sequence patterns and found new patterns in the data, we conclude that the regularities in the data are captured to a large extent by our dialogue model. This proves the suitability of the model. Now the dialogue model is validated, it can be incorporated into a comforting ECA. 
Acknowledgements This work is funded by NWO under the Responsible Innovation (RI) program.

\section{References}

1. A. de Beyn. In gesprek met kinderen:de methodiek van de kindertelefoon. SWP, 2003.

2. B.R. Burleson and D.J. Goldsmith. Handbook of Communication and Emotion: Research, Theory, Applications, and Contexts, chapter How the Comforting Process Works: Alleviating Emotional Distress through Conversationally Induced Reappraisals, pages 245-280. Academic Press, 1998.

3. J.M. van der Zwaan, V. Dignum, and C.M. Jonker. A bdi dialogue agent for social support: Specification and evaluation method. In Proceedings of the 3rd Workshop on Emotional and Empathic Agents @ AAMAS 2012, 2012.

4. J.M. van der Zwaan, V. Dignum, and C.M. Jonker. A conversation model enabling intelligent agents to give emotional support. In Proceedings of the 25th International Conference on Industrial, Engineering and Other Applications of Applied Intelligent Systems (IEA/AIE 2012), Dalian, China, 2012. 